\title{
Defining the Temperature of an Isolated Molecule
}

\author{
Mario Barbatti \\ Aix Marseille University, CNRS, ICR, Marseille, France \\ Institut Universitaire de France, 75231 Paris, France \\ mario.barbatti@univ-amu.fr|www.barbatti.org
}

\begin{abstract}
The microcanonical temperature of an isolated molecule is derived in terms of Boltzmann and Gibbs volume entropies within the quantum harmonic vibrational and equivalent degenerated model approximations. The effects of the entropy functional choice and various approximations are examined. The difference between Boltzmann and Gibbs volume temperatures is negligible for molecules bigger than ten atoms. However, it is significant for smaller systems, opening a way to probe them experimentally. A simple, analytical expression of the temperature as a function of the vibrational energy is provided, allowing predictions with a $\pm 3 \%$ margin of error. The microcanonical temperature is discussed and exemplified with polycyclic aromatic hydrocarbon molecules and other molecules of astrophysical interest.
\end{abstract}




\section{Introduction}

Any excess of vibrational energy in a molecule above its zero-point level may induce physical-chemical reactions like isomerization, dissociation, or rearrangement. The role of such vibrational energy excess is particularly relevant when a light-excited molecule transfers the photon energy to the ground state without emitting radiation. The classic example is the vision mechanism, where a complex sequence of events is triggered in the rhodopsin protein after the retinal chromophore converts photon into vibrational energy. ${ }^{1}$

We often say that a molecule with large vibrational energy is hot. However, if we ask what the molecule's temperature is, the question sounds odd. The fundamental thermochemistry of a canonical system tells that the environmental temperature determines the vibrational excess through

$$
E\left(T_{e}\right)=E_{Z P}+\sum_{k} \frac{h v_{k}}{e^{h v_{k} / k_{B} T_{e}}-1},
$$

where $E_{Z P}$ is the vibrational zero-point energy, $T_{e}$ is the environmental temperature, $k_{B}$ is the Boltzmann constant, $h$ is the Planck constant, and the sum runs over the vibrational degrees $k$ with frequency $v_{k}$. Nevertheless, an isolated molecule cannot count on the environment to thermalize. The vibrational energy excess will be trapped there until it is irradiated or induces a chemical reaction.

A hot, isolated molecule is a microcanonical system with constant total energy, and, different from the canonical problem, we want to determine $T_{m}(E)$, the microcanonical temperature as a function of the energy. Defining the temperature of such an isolated molecule could be worth doing, ${ }^{2}$ especially if we aim at describing large systems. Indeed, the temperature of isolated molecules and small finite systems has been under scrutiny for decades in diverse fields. It is needed for computing unimolecular reaction rates, ${ }^{2-4}$ sampling initial conditions for dynamics, ${ }^{5}$ studying fragmentation and radiative cooling of electrostatically trapped ions, ${ }^{6-8}$ determining thermionic electron emission of clusters beams, ${ }^{9}$ characterizing phase transitions in clusters, ${ }^{10}$ or assigning molecular species in the interstellar medium. ${ }^{11-13}$ Indeed, defining such a temperature may be helpful even for understanding the photophysics of chromophores in vacuumlike hydrophobic cavities of proteins. ${ }^{14}$ 
On the one hand, the temperature of isolated systems has been addressed pragmatically using phenomenological approaches for applied purposes. Berry and Smirnov, ${ }^{10}$ for instance, started from a reasonable estimate for the heat capacity of isolated clusters to derive their temperature. Another common approach has been to derive the canonical temperature for a canonical ensemble with kinetic averages equivalent to those of the microcanonical system. ${ }^{3}$ Andersen, Bonderup, and Hansen ${ }^{15}$ followed this way to derive a high-energy approximation for the molecular temperature. D'Hendecourt and co-workers ${ }^{11}$ estimated the properties of molecules in the interstellar medium from the observed temperature, also using an equivalent canonical approach. Most of these works, however, either do not rest on a solid statistical basis or have a limited validity domain.

On the other hand, more statistical-mechanics-oriented works have provided much knowledge on small, isolated systems. We know, for instance, that the microcanonical temperature can be defined independently of the probe, ${ }^{16}$ the type of fluctuation we may expect, including the dependence on different entropy definitions, ${ }^{17-20}$ and how to count microstates of finite coupled systems. ${ }^{21,22}$ Nevertheless, these works usually do not allow for a practical estimate of the temperature in applied cases of interest.

This paper aims to bridge this gap between theory and applications by deriving a microcanonical temperature for an isolated molecule that can be routinely used in diverse applied fields, has an extended validity domain, and is based on solid statistical mechanics grounds.

\section{Theoretical model}

To define the microcanonical temperature of an isolated molecule, we must go back to the principles of statistical thermodynamics. For a system with total energy $E$, the temperature is defined as

$$
T=\left(\frac{\partial S}{\partial E}\right)^{-1}
$$


where $S(E)$ is the entropy, which is a function of the number of microstates at energy $E$. Later, we will discuss what functional of $E$ is the most adequate when dealing with a small system far from the thermodynamical equilibrium.

If we approximate our isolated molecule by an ensemble of $N$ quantum harmonic oscillators, the total molecular energy is

$$
E_{\xi}=E_{Z P}+\sum_{k} n_{k} h v_{k}
$$

where $n_{k}$ is the number of quanta deposited in vibrational mode $k$, and $\xi$ denotes a particular ensemble $\left\{n_{k}\right\}$. Thus, to know the number of microstates, we must determine the number of solutions of Eq. (3). This means the number of ways we can distribute different values of $n_{k}$ among the vibrational modes, keeping $E$ constant. Despite the simplicity of this formulation, it is a formidably tricky problem to solve analytically. We can approach it numerically, but the number of microstates is so large that it may become unattainable to compute even for a molecule with only six atoms.

Fortunately, as I will also show later (Section 2.5.3), we can get an approximated solution for the number of microstates by supposing the ensemble of vibrational frequencies $\left\{v_{k}\right\}$ can be replaced by a single average frequency $\bar{v}$. The degenerated problem, which simplifies to

$$
E_{M}=\left(\frac{N}{2}+M\right) h \bar{v},
$$

where $M=\sum_{k} n_{k}$, is a very well-known statistical-mechanics textbook example. ${ }^{23,24}$

\subsection{Modeling an isolated molecule}

For modeling the molecule, we assume 1) that it is rigid enough, so its vibrational modes are harmonic; 2) there are no external fields; 3 ) it populates only a single electronic state.

The first assumption implies that we will not describe strongly anharmonic modes, like intramolecular hydrogen bonds or internal rotations (like those methyl groups are prone to). We can consider an isolated molecule as an ensemble of weakly coupled quantum harmonic 
oscillators. By "weakly coupled," I mean that energy can flow between vibrational modes, but the vibrational energy is still approximately given by Eq. (3). This energy flow is crucial for the molecule to ensure that all microstates sharing the same energy are equally probable. For instance, suppose the molecule has $v_{1}=300 \mathrm{~cm}^{-1}$ and $v_{2}=600 \mathrm{~cm}^{-1}$, a microstate with $n_{1}=2$ and $n_{2}=0$ should be as likely to occur as another one with with $n_{1}=0$ and $n_{2}=1$ (assuming that $n_{k}$ for $k>2$ is the same in both microstates). Although a weak coupling is assumed, it is never explicitly considered here.

The second assumption implies that we will also not discuss temperature associated with the energy allocated in the translational and rotational modes. Note, however, that these components are relevant when probing the isolated molecule. ${ }^{25}$ The third assumption means that we will not deal with the sudden vibrational energy change observed during internal conversion.

\subsection{Reviewing the statistical mechanics' concepts}

Before proceeding with the discussion, it is worth revisiting some fundamental concepts from statical mechanics. ${ }^{18}$ The basic quantities we need are the density of states

$$
\omega(E, Z)=\operatorname{Tr}[\delta(E-H(\xi, Z))]
$$

and the integrated density of states

$$
\Omega(E, Z)=\operatorname{Tr}[\Theta(E-H(\xi, Z))]
$$

In these equations, $E$ is the energy of a system with a Hamiltonian $H(\xi, Z) . Z$ comprises all external control variables like volume or fields, and $\xi$ describes the microscopic state (in our case, $\xi$ collects the quantum numbers $n_{k}$ in Eq. (3)). In Eq. (5), $\delta$ is the Dirac function and, in Eq. (6), $\Theta$ is the unit-step function. The trace Tr denotes the integral over the Hilbert space.

The entropy can be defined in different ways. For regular systems in the thermodynamic limit $(N \rightarrow \infty)$, they all tend to converge to the same result. Nevertheless, when dealing with an isolated molecule, the number $N$ of degrees of freedom is small, and we cannot assume such equivalence. Among several possibilities, we will discuss two entropy formulations, the Boltzmann entropy ${ }^{26}$ 


$$
S_{B}(E, Z)=k_{B} \ln [\varepsilon \omega(E, Z)],
$$

and the Gibbs volume entropy ${ }^{18,26,27}$

$$
S_{G}(E, Z)=k_{B} \ln [\Omega(E, Z)]
$$

In Eq. (7), $\varepsilon$ is a small energy constant.

With either entropy formulation, the microcanonical temperature can be calculated with Eq. (2) and the heat capacity with

$$
C=\left(\frac{\partial T}{\partial E}\right)^{-1}
$$

\subsection{Counting microstates}

We can directly count the microstates contributing to either $\varepsilon \omega\left(E_{\xi}\right)$ or $\Omega\left(E_{\xi}\right)$, compute the entropy, and evaluate the derivative in Eq. (2) numerically to get the $\operatorname{Boltzmann}\left(T_{B}^{(n)}\right)$ and the Gibbs volume $\left(T_{G}^{(n)}\right)$ temperatures. The superscript $(n)$ denotes the numerical approach to distinguish from other approaches discussed below. To apply the numerical procedure, we note that the molecular energy given by Eq. (3) changes by no less than the energy of the slowest normal mode $v_{1}$. Thus, the number of microstates with energy between $E_{k}=E_{Z P}+k h v_{1}$ and $E_{k}+h v_{1}$ $(k=0,1,2 \ldots)$ is

$$
h v_{1} \omega\left(E_{k}\right)=\sum_{n_{1}} \cdots \sum_{n_{N}} E_{n_{1} \cdots n_{N}} \eta\left(E_{k}\right)
$$

where

$$
\eta\left(E_{k}\right)=\left\{\begin{array}{cc}
1 & \text { if } E_{k} \leq E_{n_{1} \cdots n_{N}}<E_{k}+h v_{1} \\
0 & \text { otherwise }
\end{array}\right.
$$

The integrated density of states is 


$$
\Omega\left(E_{k}\right)=\sum_{j=0}^{k} h v_{1} \omega\left(E_{j}\right)
$$

For more advanced strategies to count microstates considering coupled modes, see Ref. ${ }^{21}$

\subsection{Solving the degenerated problem}

As mentioned, the degenerated problem is used below (Section 2.5) as an approximation for the molecular problem. Besides that, it is also helpful to check some basic hypotheses and approximations underlying the temperature definition, namely:

- the equivalence between Gibbs volume and Boltzmann entropy;

- the extension of discrete into continuous variable needed to compute derivatives;

- the effect of Stirling's approximation, which is commonly used to simplify the results of the microstates' counting;

- the impact of the $N \gg 2$ approximation, also commonly applied to simplify the results.

In the following two subsections, we will discuss the solution of the degenerated problem in the Boltzmann and Gibbs volume formulations. Although the solution in the Boltzmann formulation discussed in the previous section is well known, ${ }^{23,}{ }^{24} \mathrm{I}$ am not aware of any demonstration using the Gibbs volume formulation so far. Subsection 2.4.3 compares both results and shows how the Boltzmann temperature to Gibbs volume temperature for large $N$.

\subsubsection{The Boltzmann formulation}

The number of microstates satisfying Eq. (4) is the number of ways of allocating $M=E_{M} / h \bar{\nu}-N / 2$ quanta in $N$ vibrational modes. Thus, the number of microstates between $E$ and $E+h \bar{v}$ is the number of multisets of length $M$ on $N$ elements:

$$
\varepsilon \omega\left(E_{M}\right)=\left(\left(\begin{array}{l}
N \\
M
\end{array}\right)\right)=\left(\begin{array}{c}
M+N-1 \\
M
\end{array}\right)=\frac{(M+N-1) !}{(N-1) ! M !},
$$


where $\left(\left(\begin{array}{c}m \\ n\end{array}\right)\right)$ and $\left(\begin{array}{c}m \\ n\end{array}\right)$ denote the number of multisets and the binomial coefficient, respectively. The dependence on $E_{M}$ is implicit in $M$. Taking $\varepsilon=h \bar{v}$ in Eq. (7) allows directly compute the Boltzmann entropy as

$$
S_{B}\left(E_{M}\right)=k_{B} \ln \left[\frac{(M+N-1) !}{(N-1) ! M !}\right]
$$

The extension of this equation from a discrete to a continuous variable ( $E_{M} \rightarrow E$ ) allows using Eq. (2) to get

$$
T_{B}^{(e)}=\left(\Psi\left(\frac{E}{h \bar{\nu}}+\frac{N}{2}\right)-\Psi\left(\frac{E}{h \bar{\nu}}-\frac{N}{2}+1\right)\right)^{-1} \frac{h \bar{\nu}}{k_{B}}
$$

where $\Psi$ is the digamma function defined as

$$
\Psi(x)=\frac{d}{d x} \ln [\Gamma(x)]
$$

and $\Gamma$ is the gamma function. The superscript $(e)$ indicates this is an extended-to-continuous approximation.

Alternatively, we can employ Stirling's approximation $\ln (n !) \approx n \ln (n)-n$ in Eq. (14). Then, with Eq. (2), we get

$$
T_{B}^{(s)}=\left(\ln \left[\frac{(2 E+(N-2) h \bar{\nu})}{(2 E-N h \bar{v})}\right]\right)^{-1} \frac{h \bar{\nu}}{k_{B}}
$$

where the superscript (s) indicates Stirling's approximation. Finally, for large systems, we have $N \gg 2$, and we get the well-known result

$$
T_{B}^{(l)}=\left(\ln \left[\frac{2 E+N h \bar{\nu}}{2 E-N h \bar{\nu}}\right]\right)^{-1} \frac{h \bar{\nu}}{k_{B}} .
$$

Schwabl ${ }^{28}$ offers an alternative approach to computing $T_{B}$. Instead of using combinatorial to estimate $\varepsilon \omega(E)$, he rewrites this quantity in terms of the Fourier transform of the Dirac function 
in Eq. (5). The resulting integral is evaluated via the Laplace method, which requires $N \rightarrow \infty$. This approximation once more yields $T_{B}^{(l)}$.

\subsubsection{The Gibbs volume formulation}

To solve the degenerated problem in the Gibbs volume formulation, we proceed along the same lines as in the previous section, but employing the integrated density of states in Eq. (6). For the quantum harmonic oscillator, this quantity is the number of microstates with energy between 0 and $E$,

$$
\begin{aligned}
\Omega\left(E_{M}\right) & =\sum_{K=0}^{M}\left(\left(\begin{array}{l}
N \\
K
\end{array}\right)\right)=\sum_{K=0}^{M}\left(\begin{array}{c}
K+N-1 \\
K
\end{array}\right) \\
& =\left(\begin{array}{c}
M+N \\
M
\end{array}\right)=\frac{(M+N) !}{N ! M !}
\end{aligned}
$$

where the sum runs over the density of states defined in Eq. (13) and is evaluated using the hockeystick identity. ${ }^{29}$ Thus, the Gibbs volume entropy is

$$
S_{G}\left(E_{M}\right)=k_{B} \ln \left[\frac{(M+N) !}{N ! M !}\right] .
$$

Replacing it in Eq. (2) gives the extended-to-continuous $\left(E_{M} \rightarrow E\right)$ approximation for the microcanonical temperature

$$
T_{G}^{(e)}=\left(\Psi\left(\frac{E}{h \bar{\nu}}+\frac{N}{2}+1\right)-\Psi\left(\frac{E}{h \bar{\nu}}-\frac{N}{2}+1\right)\right)^{-1} \frac{h \bar{v}}{k_{B}} .
$$

Alternatively, with Stirling's approximation in Eq. (20), we have

$$
T_{G}^{(s)}=\left(\ln \left[\frac{2 E+N h \bar{v}}{2 E-N h \bar{v}}\right]\right)^{-1} \frac{h \bar{v}}{k_{B}} .
$$

Replacing $T_{G}^{(s)}$ in Eq. (9) gives the heat capacity

$$
C_{G}^{(s)}=\frac{N k_{B}}{4}\left(\left(\frac{2 E}{N h \bar{v}}\right)^{2}-1\right) \ln \left(\frac{2 E+N h \bar{v}}{2 E-N h \bar{v}}\right)^{2} .
$$




\subsubsection{Comparing Boltzmann and Gibbs volume formulations}

Let us now compare the previous results for the degenerated problem for a particular example of a system composed of six quantum harmonic oscillators, all with frequency $1015 \mathrm{~cm}^{-1}$. Its number of states $\varepsilon \omega(E)$ and the integrated density of states $\Omega(E)$ are plotted in Figure 1-top. Both $\varepsilon \omega(E)$ and $\Omega(E)$ grow monotonically. The energy resolution is determined by $\varepsilon$, which for the degenerated problem is $h \bar{v}$. For typical photoexcited systems, we may expect vibrational energy excess of about 2 to $4 \mathrm{eV}$. These values are much bigger than the vibrational frequency ( $0.14 \mathrm{eV}$ in our example). Thus, we can compute the temperature by numerical differentiation of the entropy (Eq. (2)), using either $\varepsilon \omega(E)$ to get $T_{B}^{(n)}$ or $\Omega(E)$ to get $T_{G}^{(n)}$.

The diverse temperature models are illustrated in the bottom panel of Figure 1. The difference between the temperatures computed with the Boltzmann and Gibbs volume formulations is striking. Although they are similar at low energies, Boltzmann temperature grows much faster, and the difference between the two formulations at $4 \mathrm{eV}$ amounts to about $1400 \mathrm{~K}$. This divergence between Boltzmann and Gibbs volume temperatures is caused by the small size of our model, $N=6$, corresponding to a four-atoms molecule. The difference reduces quickly for bigger systems. For a molecule with 15 atoms $(N=39)$, the temperature difference at $4 \mathrm{eV}$ is only $15 \mathrm{~K}$. Indeed, in the thermodynamic limit $(N \rightarrow \infty)$, Boltzmann and Gibbs volume temperatures tend to the same values, ${ }^{17}$ as we can see comparing Eqs. (18) and (22).

We also can see in Figure 1-bottom that the extended-to-continuous approximations $T_{B}^{(e)}$ and $T_{G}^{(e)}$ using the gamma function are spot-on. Stirling's approximation in $T_{B}^{(s)}$ and $T_{G}^{(s)}$ slightly degrades the result, but its main problem is at the very low energies near the zero-point level. As long we are not dealing with this region, we may favor Stirling's approximation given the algebraic simplicity of $T_{B}^{(s)}$ and $T_{G}^{(s)}$.

In the Boltzmann formulation, it is usual to assume that $N \gg 2$, yielding $T_{B}^{(l)}$ in Eq. (18). Although this approximation is fully justified for large systems, it has a major conceptual implication: it shifts the system from a Boltzmann to a Gibbs volume description, as made evident by Eqs. (18) and (22), where we can see that $T_{B}^{(l)}=T_{G}^{(s)}$. 

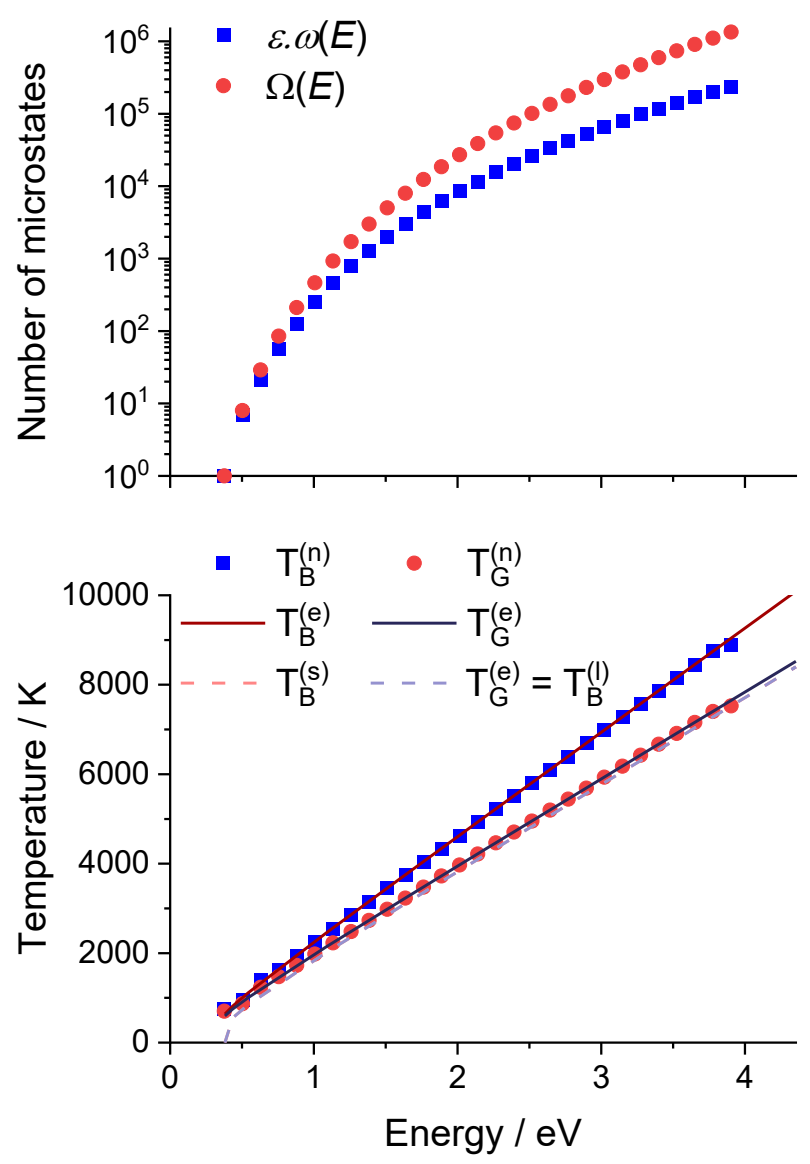

Figure 1. Top: Number of states $\varepsilon \omega(E)$ and the integrated density of states $\Omega(E)$ as a function of energy $E$ for a system composed of $N=6$ degenerated quantum harmonic oscillators of frequency $\bar{v}=1015 \mathrm{~cm}^{-1}$. Bottom: Temperature for the same degenerated system computed with different models.

Several authors, ${ }^{23,30,31}$ starting from the Boltzmann formulation, already apply the large $N$ approximation at the level of the number of microstates, Eq. (13). Because this approximation reduces Eq. (13) to Eq. (19), they inadvertently work in the Gibbs volume formulation. 


\subsection{The temperature of an isolated molecule}

\subsubsection{Defining the average frequency}

My hypothesis to compute the temperature of an isolated molecule is that there is an average frequency $\bar{v}$ for the $N$-degenerated problem that is a good approximation for the isolated molecule, composed of $N$ different frequencies. I tested two possibilities for the equivalent degenerated model (EDM), $\bar{v}$ given as the arithmetic mean

$$
\bar{v}_{A}=\frac{1}{N} \sum_{k} v_{k}=\frac{2 E_{Z P}}{N}
$$

and the harmonic mean

$$
\bar{v}_{H}=\left(\frac{1}{N} \sum_{k} \frac{1}{v_{k}}\right)^{-1} .
$$

The rationale for adopting $\bar{v}_{A}$ is the direct analogy to the solution of the degenerated problem. Note, for instance, that Eq. (22) can be rewritten as

$$
T_{G}^{(s)}=\left(\ln \left[\frac{E+E_{Z P}}{E-E_{Z P}}\right]\right)^{-1} \frac{2 E_{Z P}}{N k_{B}} .
$$

Thus, it is natural to search for an approximated solution of the non-degenerated problem with the same functional form. On the other hand, for a given $E$, there are many more microstates involving changes in the number of quanta in the low-frequency than in the high-frequency modes. Thus, it may be helpful to weigh the average frequency toward the low-frequency modes. If we take as weight the maximum number of quanta we can add to a specific vibrational mode, $n_{k}^{\max }=E / h v_{k}$ corresponding to the microstate $\left(0, \cdots, n_{k}^{\max }, \cdots, 0\right)$, we get

$$
\frac{\sum_{k} n_{k}^{\max } v_{k}}{\sum_{k} n_{k}^{\max }}=\bar{v}_{H},
$$

providing a rationale for adopting the harmonic mean of the frequencies. 
In comparing these two possibilities, we will see in Sections 2.5.2 and 2.5.3 that the arithmetic mean does better than the harmonic mean.

\subsubsection{Test case: the temperature of $\mathrm{H}_{2} \mathrm{O}_{2}$}

Let us now work out a specific example of a small, isolated molecule, $\mathrm{H}_{2} \mathrm{O}_{2}$, for which we can count the microstates and compute the temperature numerically. $\mathrm{H}_{2} \mathrm{O}_{2}$ has four atoms and $N=$ 6 vibrational modes with harmonic frequencies 346, 956, 1330, 1469, 3704, and $3709 \mathrm{~cm}^{-1}$ (computed with the geometry, frequency, noncovalent, extended tight-binding (GFN1-xTB) $\operatorname{method}^{32}$ ). The arithmetic mean frequency is $\bar{v}_{A}=1919 \mathrm{~cm}^{-1}$, and its harmonic mean frequency is $\bar{v}_{H}=1015 \mathrm{~cm}^{-1}$. Up to $4 \mathrm{eV}$, it has 169,501 microstates, distributed as a function of $E$ as shown in Figure 2-top. The number of states $\varepsilon \omega(E)$ is computed with an energy resolution of $\varepsilon=h v_{1}(346$ $\left.\mathrm{cm}^{-1}\right)$.

The Boltzmann and Gibbs volume temperatures of the isolated $\mathrm{H}_{2} \mathrm{O}_{2}$ computed by numerical differentiation of the entropy (Eq. (2)) are shown as dots in Figure 2, middle and bottom. The approximated results using the arithmetic mean frequency in the 6-degenerated problem are shown in the middle and the harmonic mean in the bottom figure. For a vibrational energy excess of $3.9 \mathrm{eV}$, the Boltzmann temperature reaches about $9013 \mathrm{~K}$ and the Gibbs volume, $7482 \mathrm{~K}$.

The growth of the number of states $\varepsilon \omega(E)$ with $E$, although monotonic, shows significant fluctuations, especially in the low-energy region. Consequently, the numerically-computed Boltzmann temperature $\left(T_{B}^{(n)}\right.$ in Figure 2) also fluctuates around the 6-degenerated mean value. The integrated density of states $\Omega(E)$ washes out most of these fluctuations and yields a much smoother Gibbs volume temperature function $\left(T_{G}^{(n)}\right)$.

All approximated results are excellent in the region above $1 \mathrm{eV}$. The agreement deteriorates in the low-energy region near the zero-point energy. The exception is Stirling's approximation with arithmetic mean frequency, which also describes the low energy well. The extended-tocontinuous approximation does not hold the correct behavior at low energies, delivering too hightemperature predictions. 

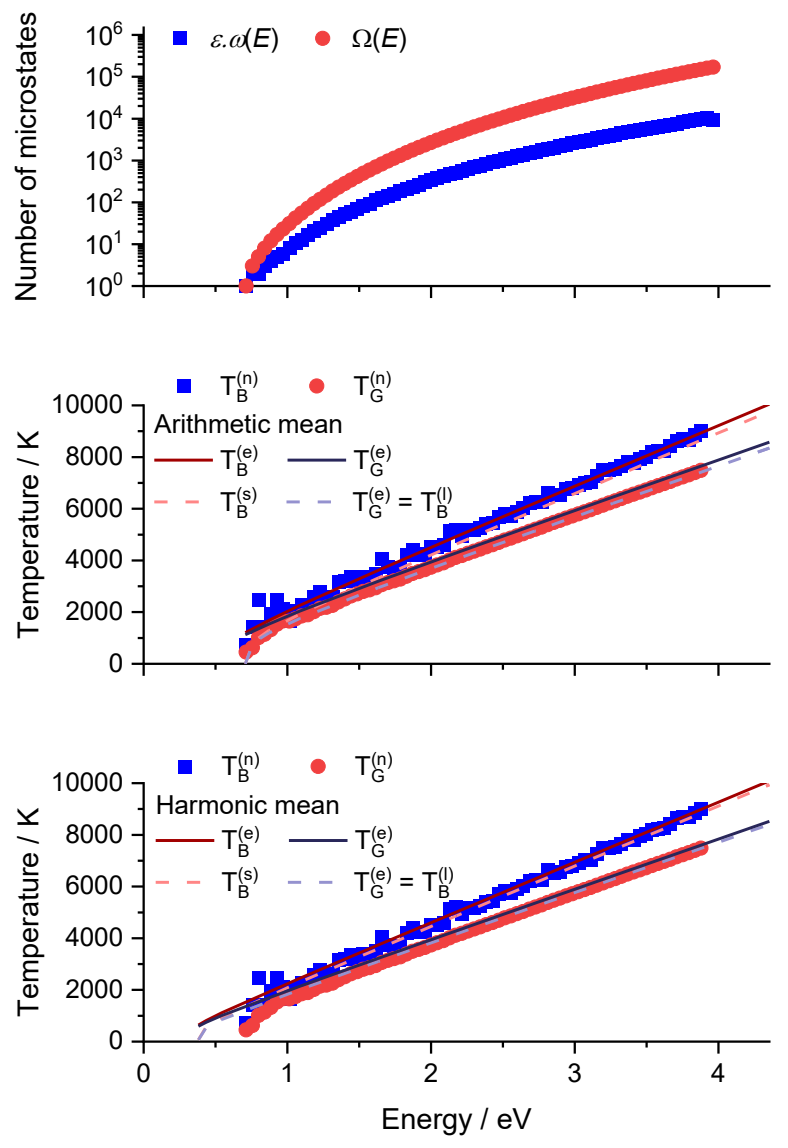

Figure 2. Top: Number of states $\varepsilon \omega(E)$ and the integrated density of states $\Omega(E)$ as a function of energy $E$ for $\mathrm{H}_{2} \mathrm{O}_{2}$. Middle: Temperature of the isolated $\mathrm{H}_{2} \mathrm{O}_{2}$ (dots). The lines are the temperatures for the $N=6$ equivalent degenerated model (EDM) with the arithmetic mean frequency of $\mathrm{H}_{2} \mathrm{O}_{2}, \bar{v}=1919 \mathrm{~cm}^{-1}$. Bottom: Temperature of the isolated $\mathrm{H}_{2} \mathrm{O}_{2}$ (dots). The lines are the temperatures for the $N=6 \mathrm{EDM}$ with the harmonic mean frequency of $\mathrm{H}_{2} \mathrm{O}_{2}, \bar{v}=1015 \mathrm{~cm}^{-1}$.

\subsubsection{Model validation}

The equivalente degenerated model with arithmetic mean frequency gave an excellent approximation for the temperature of the isolated $\mathrm{H}_{2} \mathrm{O}_{2}$. Nevertheless, is this a general result or just a coincidence? To check this point, I have computed the Gibbs volume temperature for 30 arbitrary systems (10 with $N=6,10$ with $N=7$, and 10 with $N=8$ ) with frequencies randomly distributed between 300 and $3000 \mathrm{~cm}^{-1}$. The systems with $N$ equal 7 and 8 do not correspond to 
molecules because $N$ must be $3 N_{a t}-6$. Nevertheless, they are useful to assess the errors. The systems with $N=8$ have about $10^{9}$ microstates up to $4 \mathrm{eV}$ (see Eq. (19)). $N=9$, which correspond to $N_{a t}=5$, is already unaffordable. For each energy value, the relative error

$$
\delta T=\left|\frac{T_{B / G}^{(n)}-T_{B / G}^{(s)}\left(\bar{v}_{H / A}\right)}{T_{B / G}^{(n)}}\right|
$$

between the numerical $T_{B}^{(n)}$ and $T_{G}^{(n)}$ temperatures and the temperatures of the equivalent degenerated model using Stirling's approximation was evaluated. These errors are shown in Figure 3 for temperatures computed with arithmetic mean frequencies.

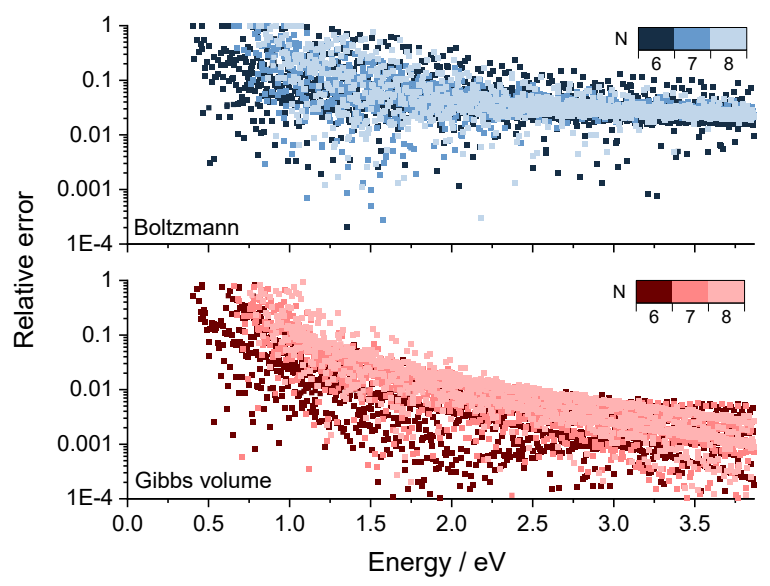

Figure 3. Relative error $\delta T$ between the numerical temperature and the Stirling's approximated temperature of the equivalent degenerated model with arithmetic mean frequencies computed for 30 random systems with $N=6$, 7, and 8. Top: Boltzmann; bottom: Gibbs volume.

Most of the error is in the low-energy region, and they are independent of the system size. The mean relative errors in Table 1 confirm this picture. $T_{G}^{(s)}$ with arithmetic mean frequency has a mean relative error of $3 \%$ no matter $N$. The mean relative error of $T_{B}^{(s)}$ also with arithmetic mean frequency is a bit bigger, $8 \%$. The mean relative errors with harmonic means are always larger than those with arithmetic mean. They are also dependent on the system size, reducing for bigger $N$. It is not shown in the table, but most of the error when using the harmonic mean frequency is 
in the low-energy region. Given the better performance of $\bar{v}_{A}$ over $\bar{v}_{H}$, only the former will be used in the next sections.

Table 1. Mean relative error $\langle\delta T\rangle$ between numerical and EDM approximated temperatures for Gibbs volume $(\mathrm{G})$ and Boltzmann $(\mathrm{B})$ formulations with harmonic $(\mathrm{H})$ and arithmetic $(\mathrm{A})$ mean frequencies.

\begin{tabular}{cccc}
\hline & $N=6$ & $N=7$ & $N=8$ \\
\hline$\left\langle\delta T_{G H}\right\rangle$ & 0.24 & 0.14 & 0.16 \\
$\left\langle\delta T_{G A}\right\rangle$ & 0.03 & 0.03 & 0.03 \\
$\left\langle\delta T_{B H}\right\rangle$ & 0.29 & 0.16 & 0.11 \\
$\left\langle\delta T_{B A}\right\rangle$ & 0.08 & 0.08 & 0.08 \\
\hline
\end{tabular}

\section{Discussion}

\subsection{Leading terms in the molecular temperature}

The asymptotic expansion of $T_{G}^{(s)}$ (Eq. (22)) at $E \rightarrow \infty$ gives

$$
T_{G}^{(a)}=\frac{E}{N k_{B}}-\frac{N h^{2} \bar{v}^{2}}{12 k_{B} E}+O\left(\frac{1}{E^{3}}\right)
$$

The leading term is linear in $E$ and does not depend on the particularities of the molecule, only on its size through $N$.

In cluster studies, it is common to estimate the microcanonical temperature using the phenomenological formula ${ }^{10,33}$

$$
T_{\text {cluster }} \approx \frac{2\left\langle E_{\text {kin }}\right\rangle}{N k_{B}}
$$

where $\left\langle E_{k i n}\right\rangle$ is the mean kinetic energy of the cluster. For a harmonic system, $2\left\langle E_{k i n}\right\rangle=E$ and, therefore, Eq. (30) corresponds to the leading term in the Gibbs volume temperature expansion, Eq. (29). 
The isolated molecule modeled developed here is purely based on a harmonic approximation. Belega and co-workers ${ }^{33}$ showed that anharmonic effects could be included in Eq. (30) in by replacing $N$ by an effective $N_{\text {eff }}$ factor computed from the reduced averaged kinetic energy in each mode.

If, instead of the Gibbs volume, we start from the Boltzmann temperature $T_{B}^{(s)}$ (Eq. (17)), the asymptotic expansion at $E \rightarrow \infty$ gives

$$
T_{B}^{(a)}=\frac{E-\frac{1}{2} h \bar{v}}{(N-1) k_{B}}-\frac{(N-1) h^{2} \bar{v}^{2}}{12 k_{B} E}+O\left(\frac{1}{E^{2}}\right)
$$

As expected, $T_{B}^{(a)} \rightarrow T_{G}^{(a)}$ when $N \rightarrow \infty$. However, for small systems, the leading term depends on the molecular properties through $\bar{v}$. Moreover, the $(N-1)$ term in the denominator causes $T_{B}^{(a)}$ grows faster than $T_{G}^{(a)}$ with the energy.

Andersen, Bonderup, and Hansen ${ }^{15}$ derived the microcanonical temperature of a small isolated system starting from the Boltzmann entropy and searching for the average energy of a canonical system equilibrated at the microcanonical temperature. Their result for an ensemble of $N$ (non-degenerated) quantum harmonic oscillators in the high-temperature limit is

$$
T_{A B H}=\frac{E}{(N-1) k_{B}} .
$$

Considering that in this limit $E \gg h \bar{v}$, their formula matches the leading order of the $T_{B}^{(s)}$ expansion in Eq. (31).

A common assumption in phenomenological models is that the heat capacity (Eq. (9)) of the isolated molecule does not depend on the temperature, so a simple linear relation $E=C T+E_{0}$ holds between energy and temperature. ${ }^{2,8}$ According to Eq. (29), such hypothesis is satisfied for a molecule with $N$ vibrational degrees if

$$
E \gg \frac{N h \bar{v}}{2 \sqrt{3}}=\frac{E_{Z P}}{\sqrt{3}} .
$$


Note that the radiative cooling should bring the molecule to the nonlinear region even if this relation is initially satisfied.

\subsection{Experimental comparison between Boltzmann and Gibbs volume}

Currently, there is an ongoing debate on which function is the correct way of computing

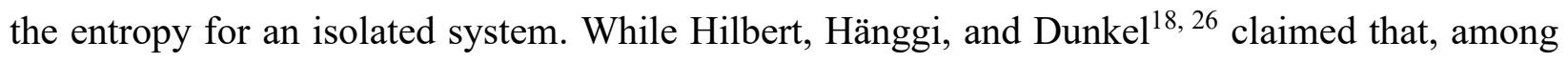
several entropy models, only Gibbs volume fulfils all three laws of thermodynamics simultaneously, Swendsen and Wang ${ }^{19}$ argued that Gibbs volume fails to satisfy the postulates of thermodynamics and make incorrect predictions for systems with nonmonotonic energy densities. (See Ref. ${ }^{34}$ for an account of this debate.)

Although this discussion goes beyond the scope of this paper, the present results create a way for experimentally testing which entropy, Boltzmann or Gibbs volume, delivers the best theoretical prediction. We could think of a setup where a small, isolated molecular ion is photoexcited in an electrostatic ion trap..$^{4,65}$ The laser pulse duration should be such as it allows thermal equilibration before radiative decay starts, but without losing too much energy resolution. Then, monitoring its radiative cooling would inform on its peak temperature (before any cooling). As discussed in Section 2.5.2, for a four-atoms molecule with a few eV of energy excess, the difference between Boltzmann and Gibbs volume temperatures should be significant enough to cause different color emissions.

\subsection{Temperature of isolated molecules of applied interest}

The temperatures $T_{B}^{(s)}$ and $T_{G}^{(s)}$ as a function of the excitation energy $\Delta E=E-E_{Z P}$ are shown in Figure 4-top for a few molecules (GFN1-xTB level). They are all systems of astrochemical interest. The difference between Boltzmann and Gibbs volume temperatures is negligible for any system above 24 vibrational degrees (10 atoms). For isolated $\mathrm{C}_{60}$ at $808 \mathrm{~K}$, Deng and $\mathrm{Echt}^{36}$ measured its heat capacity as $12.6 \pm 1.4 \mathrm{meV} / \mathrm{K}$. According to Eq. (22), this temperature corresponds to $E=14.7 \mathrm{eV}$, yielding a theoretical estimate of $C_{G}^{(s)}=12.0 \mathrm{meV} / \mathrm{K}$ (Eq. (23)), in 
excellent agreement with the experiment. Note that this result is smaller than the high-energy limit, $N k_{B}=15.0 \mathrm{meV} / \mathrm{K}(N=174)$, due to the nonlinear dependence of the temperature on the energy.

If no chemical process occurs, the hot, isolated molecule is expected to cool down due to blackbody radiation. ${ }^{6-8}$ Since the seminal papers by Sellgren ${ }^{37}$ and Leger and Puget, ${ }^{12}$ some specific near-infrared (IR) spectral features of interstellar dust have been assigned to polycyclic aromatic hydrocarbons (PAHs) ${ }^{38}$ These earlier works raised the hypothesis that those IR features arise from small, 5-10 $\AA$ molecules instantaneously heated to about $1000 \mathrm{~K}$ by UV radiation. Working on observational data, Leger and co-authors ${ }^{11,12}$ estimated a color temperature of $600 \mathrm{~K}$, corresponding to a peak temperature of $950 \pm 150 \mathrm{~K}$. Then, using the Einstein heat capacity model and assuming 6-eV photoexcitation, they predicted that the IR features should be due to carbon species with about 60 atoms.

We can revisit this assignment by using the microcanonical temperature derived here. Let us assume a peak temperature of $T=950 \pm 150 \mathrm{~K}$ and that the arithmetic mean frequency is $\bar{v}=$ $1100 \pm 100 \mathrm{~cm}^{-1}$. Let us also allow a broader variation in the UV excitation, $\Delta E_{U V}=5 \pm 1 \mathrm{eV}$ Using Eq. (22), the number of atoms is

$$
N_{a t}=\frac{\Delta E_{U V}}{3 h \bar{\nu}}\left(\exp \left(\frac{h \bar{\nu}}{k_{B} T}\right)-1\right)+2
$$

Letting all three parameters vary according to a random normal distribution with standard deviation given by the error bars, we arrive at $N_{a t}=61 \pm 38$ for $10^{6}$ random systems. This mean number of atoms agrees with the previous calculations. Nevertheless, as we can see in the density plot at the bottom of Figure 4, it also indicates that a large variety of possible molecular systems falls within the parameter constraints. The figure also shows a few PAH molecules ranging from small to large sizes. Except for naphthalene neutral and cation, they all fall in the high-density region, as we would expect from PAH mixtures that should occur in the interstellar media. ${ }^{39,40}$ This analysis considers only molecules returning ground state via radiationless pathways. If they fluoresce, the peak temperature after re-equilibration in the ground state should correspond to a vibrational excess discounting the energy of the emitted photon. 

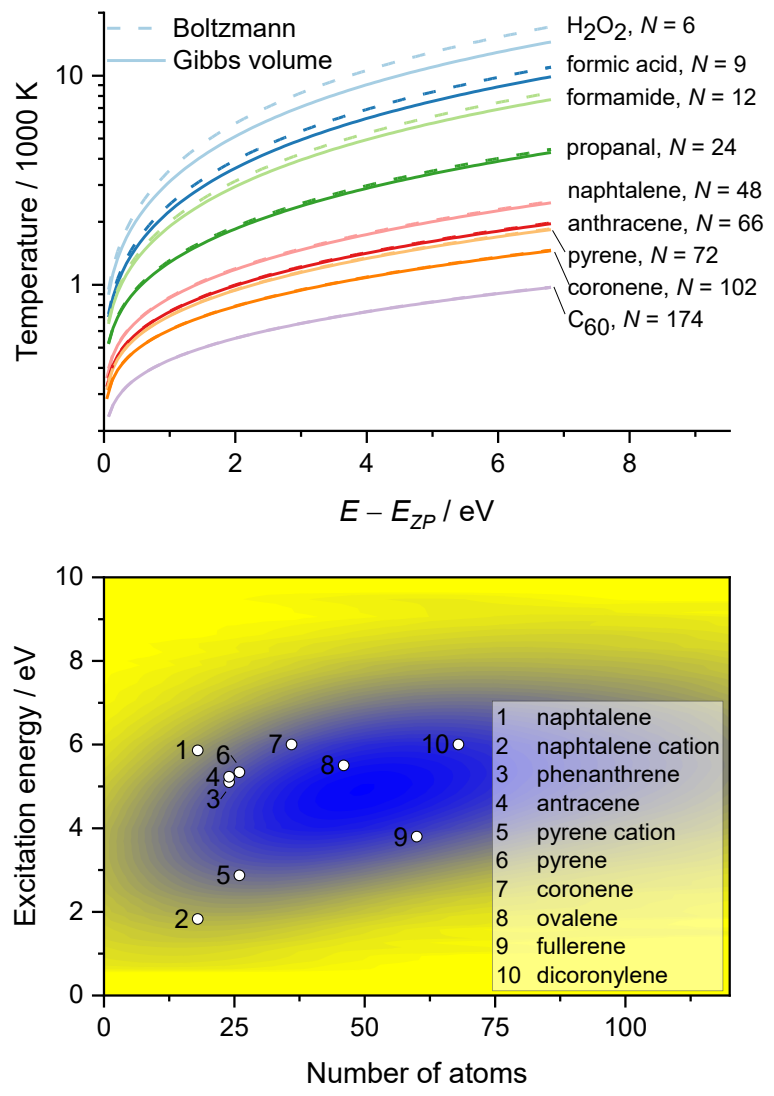

Figure 4. Top: Gibbs volume $T_{G}^{(s)}$ (solid lines) and Boltzmann $T_{B}^{(s)}$ (dashed lines) temperatures for a few molecules (arithmetic mean frequency). $N$ is the number of vibrational modes. Bottom: Density map of possible molecular systems with temperature $950 \pm 150 \mathrm{~K}$, UV excitation $5 \pm 1 \mathrm{eV}$, and arithmetic mean frequency $1100 \pm 100 \mathrm{~cm}^{-1}$. The density grows from yellow to blue. The dots illustrate the absorption peak of a few PAH molecules with excitation energies taken from the literature: anthracene, ${ }^{41}$ naphthalene (neutral and cation), phenanthrene, pyrene (neutral and cation), ${ }^{42}$ coronene, dicoronylene, ovalene, ${ }^{40}$ and fullerene. ${ }^{43}$

\section{Conclusions}

This paper explores the microcanonical temperature of an isolated molecule. The molecule is treated as an ensemble of quantum harmonic oscillators, and the temperature is derived in the 
frame of Boltzmann and Gibbs volume entropies. Although the direct solution of the problem seems not to be accessible analytically, we discuss how approximations based on an equivalent degenerated model using average frequencies can adequately describe the molecular temperature. The discussion tracks the effect of several hypotheses and approximations on the results, including the entropy functional, the type of average frequency, the extension of discrete into continuous variables, the use of Stirling's approximation, the assumption of a large number of degrees of freedom $N$, and the expansion in the leading orders. Table 2 summarizes the several expressions discussed in the text.

Table 2. Summary of the microcanonical temperatures derived under different approximations from Boltzmann and Gibbs volume entropies for a degenerated set of $N$ quantum harmonic oscillators with frequency $\bar{v}$. For the non-degenerated problem, $\bar{v}$ is the arithmetic mean frequency of the spectrum. $\Psi$ is the digamma function. For an isolated molecule wellrepresented by harmonic vibrational modes, $T_{G}^{(s)}$ delivers a good approximation for the microcanonical temperature within an error of $3 \%$.

\begin{tabular}{lll}
\hline & Boltzmann & Gibbs volume \\
\hline $\begin{array}{ll}\text { Numerical } \\
\text { counting }\end{array}$ & $T_{B}^{(n)}=\left(\frac{\Delta S_{B}}{\Delta E}\right)^{-1}$ & $T_{G}^{(n)}=\left(\frac{\Delta S_{G}}{\Delta E}\right)^{-1}$ \\
$\begin{array}{ll}\text { Extension of } E_{M} \\
\text { to continuous } E\end{array}$ & $T_{B}^{(e)}=\left(\Psi\left(\frac{E}{h \bar{v}}+\frac{N}{2}\right)-\Psi\left(\frac{E}{h \bar{v}}-\frac{N}{2}+1\right)\right)^{-1} \frac{h \bar{v}}{k_{B}}$ & $T_{G}^{(e)}=\left(\Psi\left(\frac{E}{h \bar{v}}+\frac{N}{2}+1\right)-\Psi\left(\frac{E}{h \bar{v}}-\frac{N}{2}+1\right)\right)^{-1} \frac{h \bar{v}}{k_{B}}$ \\
$\begin{array}{l}\text { Stirling's } \\
\text { approximation }\end{array}$ & $T_{B}^{(s)}=\left(\ln \left[\frac{(2 E+(N-2) h \bar{v})}{(2 E-N h \bar{v})}\right]\right)^{-1} \frac{h \bar{v}}{k_{B}}$ & $T_{G}^{(s)}=\left(\ln \left[\frac{2 E+N h \bar{v}}{2 E-N h \bar{v}}\right]\right)^{-1} \frac{h \bar{v}}{k_{B}}$ \\
$\begin{array}{l}\text { Large } N \\
\text { approximation }\end{array}$ & $T_{B}^{(l)}=\left(\ln \left[\frac{2 E+N h \bar{v}}{2 E-N h \bar{v}}\right]\right)^{-1} \frac{h \bar{v}}{k_{B}}$ & $T_{G}^{(a)}=\frac{E}{N k_{B}}-\frac{N h^{2} \bar{v}^{2}}{12 k_{B} E}+O\left(\frac{1}{E^{3}}\right)$ \\
$\begin{array}{l}\text { Asymptotic } \\
\text { expansion at }\end{array}$ & $T_{B}^{(a)}=\frac{E-\frac{1}{2} h \bar{v}}{(N-1) k_{B}}-\frac{(N-1) h^{2} \bar{v}^{2}}{12 k_{B} E}+O\left(\frac{1}{E^{2}}\right)$ & $T_{G}^{(c)}=\frac{E}{N k_{B}}$ \\
$\begin{array}{l}\text { Constant heat } \\
\text { capacity }\end{array}$ & $T_{B}^{(c)}=\frac{E-\frac{1}{2} h \bar{v}}{(N-1) k_{B}}$ & \\
\hline
\end{tabular}

The comparison between Boltzmann and Gibbs volume approaches shows that the temperatures converge to the same values for large $N$. The difference is already negligible for a 
10-atoms molecule. However, for smaller molecules, the difference is significant, and it should be possible to probe it experimentally. In particular, we discuss how a common numerical approximation corresponds, in fact, to a shift from the Boltzmann into the Gibbs volume formulation.

We also see how the microcanonical temperature relates to previous phenomenological approaches. For a large vibrational energy excess, the common assumption of a linear relationship between temperature and energy (constant heat capacity) is valid. Nevertheless, dissociative and radiative cooling should bring the system to nonlinear regions.

For practical purposes, for a molecule with more than 10 atoms, I recommend using $T_{G}^{(s)}$

(the Gibbs volume temperature $T_{G}^{(s)}$ with Stirling's approximation) or equivalently $T_{B}^{(l)}$ (the Boltzmann temperature for large $N$ ) and arithmetic mean of frequencies, which should predict temperatures within $\pm 3 \%$ margin of error. For smaller molecules, the temperature can be computed with either $T_{G}^{(s)}$ or $T_{B}^{(s)}$. At this point, it is unclear which one delivers the correct answer.

We should also bear in mind that the microcanonical temperature models discussed here are strictly valid for molecules that are not subjected to external fields, populate a single electronic state, and whose vibrational modes can be well-represented by a harmonic approximation. There are two advantages of working within these boundaries. First, they provide simple analytical expressions that allow a straightforward temperature estimation beyond the classical regime. Second, they enable the development of a conceptual understanding that is sometimes hidden when applying advanced numerical approaches. Nonetheless, the present modeling should be extended beyond these approximations in the future. The primary goal should be to tackle anharmonic systems to predict the temperature of more general systems, like molecular clusters.

\section{Acknowledgements}

MB thanks the funding provided by European Research Council (ERC) Advanced grant SubNano (Grant agreement 832237). The author is grateful to Prof. A. Verga, who brought Schwabl's alternative derivation of $T_{B}$ to his attention. 


\section{References}

${ }^{1}$ S. Gozem, H. L. Luk, I. Schapiro, and M. Olivucci, Chem. Rev. 117 (2017) 13502.

${ }^{2}$ K. Hansen, Chem. Phys. Lett. 620 (2015) 43.

${ }^{3}$ C. E. Klots, J. Chem. Phys. 90 (1989) 4470.

${ }^{4}$ K. Hansen, Mass Spectrom. Rev. 40 (2021) 725.

${ }^{5}$ R. Crespo-Otero, and M. Barbatti, Chem. Rev. 118 (2018) 7026.

${ }^{6}$ Y. Toker, O. Aviv, M. Eritt, M. L. Rappaport, O. Heber, D. Schwalm, and D. Zajfman, Phys. Rev. A 76 (2007) 053201.

${ }^{7}$ H. Zettergren, A. Domaracka, T. Schlathölter, P. Bolognesi, S. Díaz-Tendero, M. Łabuda, S. Tosic, S. Maclot, P. Johnsson, A. Steber, D. Tikhonov, M. C. Castrovilli, L. Avaldi, S. Bari, A. R. Milosavljević, A. Palacios, S. Faraji, D. G. Piekarski, P. Rousseau, D. Ascenzi, C. Romanzin, E. Erdmann, M. Alcamí, J. Kopyra, P. Limão-Vieira, J. Kočišek, J. Fedor, S. Albertini, M. Gatchell, H. Cederquist, H. T. Schmidt, E. Gruber, L. H. Andersen, O. Heber, Y. Toker, K. Hansen, J. A. Noble, C. Jouvet, C. Kjær, S. B. Nielsen, E. Carrascosa, J. Bull, A. Candian, and A. Petrignani, Eur. Phys. J. D 75 (2021) 152.

${ }^{8}$ E. Kolodney, B. Tsipinyuk, and A. Budrevich, J. Chem. Phys. 102 (1995) 9263.

${ }^{9}$ B. A. Collings, A. H. Amrein, D. M. Rayner, and P. A. Hackett, J. Chem. Phys. 99 (1993) 4174.

${ }^{10}$ R. S. Berry, and B. M. Smirnov, Phys.-Uspekhi 52 (2009) 137.

${ }^{11}$ L. D'Hendecourt, A. Léger, P. Boissel, and F. Désert, in Proceedings of the 135th Symposium of the International Astronomical Union, edited by L. J. Allamandola, and A. G. G. M. Tielens (Springer, 1989), pp. 207.

${ }^{12}$ A. Leger, and J. L. Puget, Astron. Astrophys. 500 (1984) 279.

${ }^{13}$ C. Leidlmair, P. Bartl, H. Schöbel, S. Denifl, M. Probst, P. Scheier, and O. Echt, Astrophys. J. 738 (2011) L4.

${ }^{14}$ L. H. Andersen, and A. V. Bochenkova, Eur. Phys. J. D 51 (2009) 5.

${ }^{15}$ J. U. Andersen, E. Bonderup, and K. Hansen, J. Chem. Phys. 114 (2001) 6518.

${ }^{16}$ J. Wang, and W.-g. Wang, Phys. Rev. E 96 (2017) 032207.

${ }^{17}$ L. Ferrari, Eur. Phys. J. Plus 132 (2017) 487.

${ }^{18}$ S. Hilbert, P. Hänggi, and J. Dunkel, Phys. Rev. E 90 (2014) 062116.

${ }^{19}$ R. H. Swendsen, and J.-S. Wang, Phys. Rev. E 92 (2015) 020103. 
${ }^{20}$ R. Franzosi, J. Stat. Mech. 2019 (2019) 083204.

${ }^{21}$ C. Aieta, F. Gabas, and M. Ceotto, J. Phys. Chem. A 120 (2016) 4853.

${ }^{22}$ G. Mandelli, C. Aieta, and M. Ceotto, J. Chem. Theory Comput. 18 (2022) 623.

${ }^{23}$ H. B. Callen, Thermodynamics and an Introduction to Thermostatistics (John Wiley \& Sons, 1985),

${ }^{24}$ J. E. Mayer, and M. G. Mayer, Statisical Mechancs (John Wiley Sons, Inc., 1966),

${ }^{25}$ T. M. Fuchs, F. Rivic, and R. Schäfer, Phys. Rev. A 104 (2021) 012820.

${ }^{26}$ J. Dunkel, and S. Hilbert, Nature Physics 10 (2014) 67.

${ }^{27}$ J. W. Gibbs, Elementary Principles in Statistical Mechanics (Chapter XIV) (Scribner's sons, 1902),

${ }^{28}$ F. Schwabl, Statistical mechanics (Springer Science \& Business Media, 2006),

${ }^{29}$ C. H. Jones, Fibonacci Q. 34 (1996) 280.

${ }^{30}$ P. G. Nelson, Foundations of Chemistry 21 (2019) 325.

${ }^{31}$ D. V. Schroeder, An introduction to thermal physics (Addison Wesley Longman, 2000),

${ }^{32}$ S. Grimme, C. Bannwarth, and P. Shushkov, J. Chem. Theory Comput. 13 (2017) 1989.

${ }^{33}$ E. D. Belega, E. A. Cheremukhin, P. V. Elyutin, and D. N. Trubnikov, Chem. Phys. Lett. 496 (2010) 167.

${ }^{34}$ R. H. Swendsen, Rep. Prog. Phys. 81 (2018) 072001.

${ }^{35}$ S. Martin, J. Bernard, R. Brédy, B. Concina, C. Joblin, M. Ji, C. Ortega, and L. Chen, Phys. Rev. Lett. 110 (2013) 063003.

${ }^{36}$ R. Deng, and O. Echt, J. Phys. Chem. C 119 (2015) 11233.

${ }^{37}$ K. Sellgren, Astrophys. J. 277 (1984) 623.

${ }^{38}$ M. S. Murga, M. S. Kirsanova, D. S. Wiebe, and P. A. Boley, Mon. Not. R. Astron. Soc. 509 (2021) 800 .

${ }^{39}$ M. Steglich, C. Jäger, G. Rouillé, F. Huisken, H. Mutschke, and T. Henning, Astrophys. J. 712 (2010) L16.

${ }^{40}$ G. Malloci, G. Mulas, and C. Joblin, Astron. Astrophys. 426 (2004) 105.

${ }^{41}$ A. Thöny, and M. J. Rossi, J. Photochem. Photobiol. A 104 (1997) 25.

${ }^{42}$ T. M. Halasinski, F. Salama, and L. J. Allamandola, Astrophys. J. 628 (2005) 555.

${ }^{43}$ B. B. Brady, and E. J. Beiting, J. Chem. Phys. 97 (1992) 3855. 
